\title{
Comparative Study of Conventional Ziehl Neelsen and Modified Bleach Method of Ziehl Neelsen with CBNAAT in the Diagnosis of Tuberculous Lymphadenitis
}

\author{
Gayathri Purushothaman'1, Manjaris Sekar², Saravanan Balaraman³
}

\begin{abstract}
${ }^{1}$ Department of Pathology, Tirunelveli Medical College, Tirunelveli, Tamilnadu, India. ${ }^{2}$ Vellore Medical College, Vellore, Tamilnadu, India. ${ }^{3}$ Department of Pathology, GAAMCH Research Institute, Kanchipuram, Tamilnadu, India.
\end{abstract}

\section{ABSTRACT}

\section{BACKGROUND}

Tuberculosis is one of the major health problems in developing countries like India. Even though lungs are the commonly involved organs in Tuberculosis, extrapulmonary presentations are on the rise nowadays. Among the various types of presentations of extrapulmonary tuberculosis, lymphadenitis is the most common presentation. A spectrum of investigations is available for diagnosis, and molecular methods like CBNAAT and PCR analysis are highly reliable. But their disadvantage is their cost and requirement of trained personnel. FNA is the first line of investigation but is lacking in sensitivity and culture method is time consuming. So, Modified Ziehl Neelsen method with low cost and less time may be considered as an alternative. We wanted to evaluate the sensitivity of the Modified Bleach Ziehl Neelsen method and compare the Modified and Conventional Ziehl Neelsen Method in association with the CBNAAT in the diagnosis of TB lymphadenitis.

\section{METHODS}

Diagnostic validation study was conducted with 30 clinically suspected cases of tuberculosis. FNA was done and aspirated material was spread on 2 slides. Slides were stained with $\mathrm{H} \& \mathrm{E}$ stain and convention Ziehl Neelsen stain. Part of the aspirate material was centrifuged with $5 \%$ sodium hypochlorite. Sediment was smeared on a slide and stained with Ziehl Neelsen stain. Remaining material was subjected to CBNAAT assay.

\section{RESULTS}

Correlation shows that 9,6 , and 11 out of 30 cases were positive for tuberculosis in Conventional, Modified and CBNAAT methods respectively. Sensitivity was $81.81 \%$; specificity was $94.74 \%$, positive predictive value was $90 \%$ and negative predictive value was $90 \%$ for Conventional Ziehl Neelsen Method and 54.55\%, 94.73\%, $85.71 \%$ and $78.26 \%$, respectively for modified bleach method.

\section{CONCLUSIONS}

Conventional method is found to be more sensitive than modified method. CBNAAT negative results do not rule out TB. So, they should be correlated with cytology and microbiological studies.

\section{KEY WORDS}

Tuberculosis, Ziehl Neelsen Stain, Cartridge Based Nucleic Acid Amplification Test [CBNAAT], Fine Needle Aspiration [FNA]
Corresponding Author: Dr. Saravanan Balaraman, No. 10, $1^{\text {st }}$ Street, Lakshmipuram Extension, Mudichur Road, West Tambaram, Chennai-45, Tamilnadu, India. E-mail:drsarub@gmail.com

DOI: $10.14260 /$ jemds/2020/255

Financial or Other Competing Interests: None.

How to Cite This Article: Purushothaman G, Sekar M, Balaraman S. Comparative study of conventional ziehl neelsen and modified bleach method of ziehl neelsen with CBNAAT in the diagnosis of tuberculous lymphadenitis. J. Evolution Med. Dent. Sci. 2020;9(14):1175-1179, DOI: $10.14260 /$ jemds/2020/255

Submission 29-01-2019,

Peer Review 14-03-2020,

Acceptance 21-03-2020,

Published 06-04-2020.

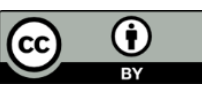




\section{BACKGROUND}

India is classified along sub Saharan African countries to be among those with high burden of Tuberculosis. ${ }^{[1]}$ As per WHO global Tuberculosis report 2015, out of the estimated global annual incidence of 9.6 million TB cases, 2.2 million were estimated to occur in India. The prevalence of TB estimated in 2013 was 195 per 100,000 persons. [2] Every day more than 20,000 people become infected with tubercle bacillus, more than 5000 develop disease and more than 1000 die from Tuberculosis.[3] Tuberculosis is caused by Mycobacterium tuberculosis complex that can affect any part of the body with lungs being the most common organ involved. Extrapulmonary tuberculosis constitutes 15 to $20 \%$ all cases of TB. $\left.{ }^{4}\right]$ Its prevalence In India varies between $8.3-13.1 \%$ in different districts.[5] Tuberculous lymphadenitis is the most common Extrapulmonary manifestations of Tuberculosis[6] and it constitutes $35 \%$ of Extrapulmonary TB which comprises 15 to $20 \%$ of all cases of TB.[7] Tuberculous lymphadenitis most commonly affects cervical lymph nodes. ${ }^{[8]}$ The peak age range was 30 to 40 years. TB lymphadenitis is most common among women than men in a ratio of $14: 1$ a different pattern akin to pulmonary tuberculosis. ${ }^{[9]}$

Various diagnostic methods of Tuberculous are Fine needle aspiration cytology, Ziehl Neelsen and modified Ziehl Neelsen method, Auramine Rhodamine fluorescent dye, culture, automated methods, molecular methods like Polymerase chain reaction and Cartridge based Nucleic acid amplification test [CBNAAT]. FNA has emerged as a first line diagnostic technique.[10,11] Nodal Tuberculosis can be diagnosed by evaluation of cytomorphological features like presence of granuloma, caseous necrosis and Langhans giant cells in fine needle aspiration cytology. The presence of cellular debris may hinder the morphology of granuloma. FNAC provides an alternative to excision biopsy for lymph nodes and is an easy procedure for collection of material for cytomorphological and bacteriological examination. There is a major role of FNAC in TB lymphadenitis diagnosis as it is a less invasive procedure and Ziehl Neelsen (ZN) method for AFB plays a key role in diagnosis and also for monitoring the treatment in Tuberculosis. In developing countries like India, one of the widely available methods for diagnosing extra pulmonary TB is microscopic smear study from lesion for AFB using ZN method but, it has low sensitivity.[12] Microscopy has many advantages when it comes to speed and feasibility. If the sensitivity could be improved, it has the potential to become a more valuable tool for TB control programme around the world.[13] Conventional Ziehl Neelsen can be used in smears to detect Acid fast bacilli. The major disadvantage of the conventional Ziehl Neelsen is its sensitivity ranging from $20 \%$ to $43 \% .^{[14,15]}$ In the last decade many researchers have suggested that the performance can be significantly improved if the sample is liquefied with one or other chemical reagents and then concentrated by centrifugation or sedimentation before acid fast staining.[16] There are various concentration methods for improving sensitivity of direct microscopy. Among these, the bleach concentration method is one of the safest to improve the sensitivity.[13]

Early diagnosis of $\mathrm{TB}$ and initiating optical treatment would not only enable a cure of individual patient but will curb transmission of infection. M. tuberculosis can also be diagnosed by culture in various medium, but it takes two to eight weeks for the colonies to appear. Several automated cultures have been developed such as BACTEC 460, BACT Alert,[17] to indicate growth rapidly, but they are not cost effective. CB-NAAT [Gene X pert] is an automated cartridgebased technique which not only detects $M$. tuberculosis but also rifampicin resistance within 2 hours. It is the first fully automated bench top cartridge based nucleic acid amplification (CBNAAT) assay for TB detection that includes all necessary steps of DNA PCR.[18] WHO recommended that the test should be used as the initial diagnostic test in individuals suspected of having MDR TB and in smear negative specimen. The main advantages of the test are reliability and the speed of getting the result.[19] However both Molecular methods and Rapid molecular assay Cartridge Based Nucleic Acid Amplification Testing (CBNAAT) are not readily available in all sectors and are costly. So to increase the diagnostic accuracy of TB lymphadenitis, a low cost, less time consuming method is needed. The bleach method of Ziehl Neelsen can fulfil the above said criteria and is economic, easily available test compared to CBNAAT.

We wanted to evaluate the sensitivity of the Modified Bleach Ziehl Neelsen method and compare the Modified and Conventional Ziehl Neelsen Method in association with the CBNAAT in diagnosis of TB lymphadenitis.

\section{METHODS}

This diagnostic validation study was conducted after obtaining approval from the institutional scientific ethical committee. Patients were selected on the basis of set of inclusion and exclusion criteria.

\section{Inclusion Criteria}

1. Patients clinically suspected of having TB lymphadenitis.

2. Patients of all ages.

\section{Exclusion Criteria}

1. Patients who are already diagnosed as TB.

2. Patients who are on anti-TB drugs.

3. Patients already treated with anti-TB drugs.

30 Patients with clinical diagnosis of TB lymphadenitis were subjected to Fine Needle Aspiration (FNA) using a 23gauge needle and one smear was fixed in $90 \%$ alcohol for 20 minutes and subsequently stained with Haematoxylin and Eosin stain. Another smear was subjected to conventional Ziehl Neelsen staining. A part of the material mixed with $2 \mathrm{ml}$ of $5 \%$ sodium hypochlorite in a clean glass test tube. The test tube was left for 20 minutes at room temperature for incubation. The specimen was centrifuged at $3000 \mathrm{rpm}$ for 15 minutes. Supernatant was discarded and sediments were taken on a clean glass slide, air dried, heat fixed and stained with routine Ziehl Neelsen stain. Slides were assessed for cytomorphology and AFB bacilli. Reactive lymphadenitis was diagnosed when the smear showed polymorphous population of lymphocytes along with tingible body macrophages. The diagnosis of suppurative lymphadenitis were made when the 
majority of cellularity composed of polymorphs along with lymphocytes, histiocytes and other inflammatory cells with or without necrotic background.

Cases of tuberculous lymphadenitis (TB lymphadenitis) were diagnosed with the following criteria-

1. Epithelioid cells with caseating necrosis.

2. Epithelioid cells without caseating necrosis.

Remaining material was subjected for CBNAAT assay. The Sensitivity and specificity of the Conventional ZN and modified bleach method are compared with CBNAAT assay, being a gold standard technique. Revised National Tuberculosis Control Programme (RNTCP) guidelines were followed in grading the bacilli.

\section{Statistical Analysis}

Statistical analysis was expressed in terms of Sensitivity, Specificity, Positive predictive value and Negative predictive value.

\section{RESULTS}

The present study included 30 cases of clinically suspected tuberculous lymphadenitis. Among 30 cases, the age group ranges from 2 to 85 yrs. with a mean age of 32 yrs. Out of 30 cases 28 cases the aspiration was done from cervical lymph nodes and in 2 cases from axillary lymph nodes. 2 patients presented with generalized lymphadenopathy. In cytology 6 cases $(20 \%)$ were found to be reactive lymphadenitis, 11 cases (36.7\%) were diagnosed as a suppurative lymphadenitis and 13 cases (43.3\%) were signed out as Tuberculous lymphadenitis. By conventional Ziehl Neelsen method, 9 cases showed acid fast bacilli. Out of 9 cases 6 were diagnosed as suppurative lymphadenitis and 3 were diagnosed as tuberculous lymphadenitis by cytology.

When comparing with that of CBNAAT, 9 cases were positive in conventional Ziehl Neelsen and 11 cases were positive in CBNAAT. One case which showed AFB by conventional Ziehl Neelsen method was negative by CBNAAT. In conventional Ziehl Neelsen, bacteria were identified as pink coloured, slender, irregular beaded curved bacilli. Most of the bacilli are in singles. When comparing with CBNAAT as gold standard, it yielded a sensitivity of $81.81 \%$, specificity of $94.74 \%$, positive predictive value (PPV) was $90 \%$ and negative predictive value (NPV) was $90 \%$. By modified bleach method of Ziehl Neelsen technique, 6 cases were positive for acid fast bacilli. Out of 6 cases, 5 cases were diagnosed as suppurative lymphadenitis and one case was tuberculous lymphadenitis by FNAC. AFB was seen mostly in clumps than in singles.

None of the reactive lymphadenitis showed acid fast bacilli in both conventional and modified bleach method of Ziehl Neelsen technique. When comparing with that of CBNAAT, one case which was positive in both conventional and modified bleach method, but bacilli was not detected by CBNAAT. With CBNAAT as gold standard method, modified bleach method of Ziehl Neelsen yielded $54.55 \%$ of sensitivity, specificity of $94.73 \%$, positive predictive value was $85.71 \%$ and negative predictive value was $78.26 \%$.

\begin{tabular}{|cccc|}
\hline & RNTCP & \\
& Result & Grading & No. of Fields \\
$>10 /$ field & Positive & $3+$ & 20 \\
$1-10 /$ field & Positive & $2+$ & 50 \\
$10-99 / 100$ field & Positive & $1+$ & 100 \\
$1-9 / 100$ field & Positive & scanty & 100 \\
No of AFB in 100 & Negative & 1000 \\
\hline Table 1. Revised National Tuberculosis Control Programme \\
Grading of Tuberculous Bacilli[20] \\
\hline
\end{tabular}

\begin{tabular}{|c|c|c|c|c|c|c|}
\hline \multirow{2}{*}{\multicolumn{3}{|c|}{ Cytology }} & \multicolumn{2}{|c|}{ Conventional ZN Method } & \multicolumn{2}{|c|}{ Bleach ZN Method } \\
\hline & & & Positive & $\begin{array}{l}\text { nal ZN Method } \\
\text { Negative }\end{array}$ & \multicolumn{2}{|c|}{ Positive Negative } \\
\hline Reactive & npha & enitis & 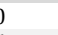 & 6 & 0 & 6 \\
\hline Suppurativ & ymph & denitis & 5 & 5 & 5 & 6 \\
\hline Tuberculou & ympl & adenitis & 3 & 10 & 1 & 12 \\
\hline \multicolumn{7}{|c|}{$\begin{array}{l}\text { Table 2. Comparison of Cytological Features with That of } \\
\text { Conventional and Bleach ZN Method }\end{array}$} \\
\hline \multicolumn{4}{|c|}{ Conventional ZN } & \multicolumn{3}{|c|}{ Bleach Method } \\
\hline Positive & $\%$ & Negative & $\%$ & Positive & Negative & $\%$ \\
\hline 9 & 30 & 21 & 70 & 20 & 24 & 80 \\
\hline \multicolumn{7}{|c|}{$\begin{array}{l}\text { Table 3. Comparison of Conventional ZN Method } \\
\text { with That of Bleach ZN Method }\end{array}$} \\
\hline
\end{tabular}

\begin{tabular}{|cccc|}
\hline & \multicolumn{3}{c|}{ CBNAAT } \\
Conventional ZN & Positive & Negative \\
Method & Positive & 9 & 1 \\
Negative & 2 & 18 \\
Comparison of Conventional ZN method with that of CBNAAT \\
\multicolumn{4}{|c|}{ CBNAAT } \\
Bleach ZN method & Positive & Negative \\
& Negative & 6 & 1 \\
Comparison of Bleach ZN method with that CBNAAT & 18 \\
\hline \multicolumn{3}{|c|}{ Table 4. Comparison of Conventional ZN Method } \\
and Bleach ZN Method with That of CBNAAT \\
\hline
\end{tabular}

\begin{tabular}{|c|c|c|}
\hline & $\begin{array}{l}\text { Conventional ZN } \\
\text { Method }\end{array}$ & $\begin{array}{l}\text { Modified Bleach } \\
\text { Method }\end{array}$ \\
\hline Sensitivity & $81.81 \%$ & $54.55 \%$ \\
\hline Specificity & $94.74 \%$ & $94.73 \%$ \\
\hline Positive predictive value & $90 \%$ & $85.71 \%$ \\
\hline Negative predictive value & $90 \%$ & $78.26 \%$ \\
\hline \multicolumn{3}{|c|}{$\begin{array}{c}\text { Table 5. Statistical Comparison between Conventional ZN } \\
\text { with Modified Bleach ZN Method }\end{array}$} \\
\hline
\end{tabular}

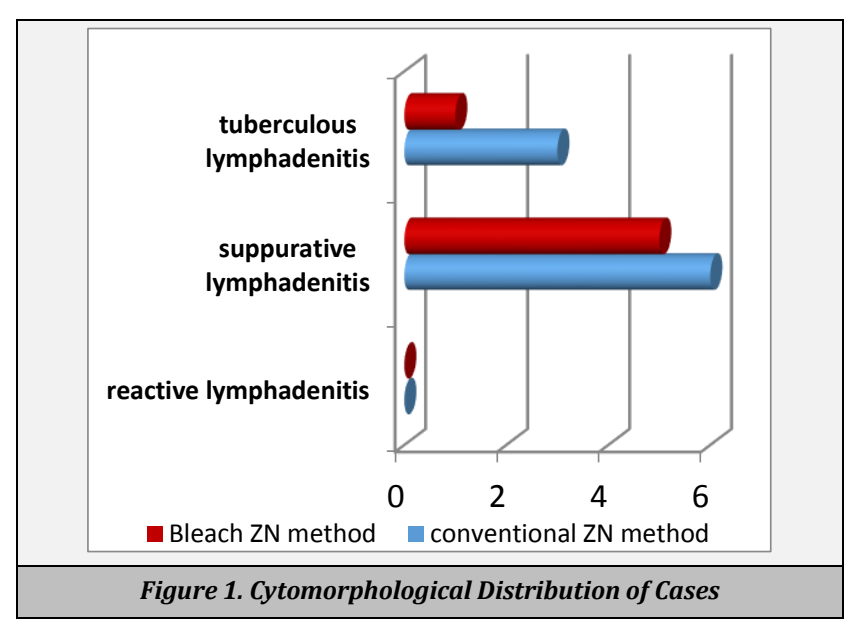

\section{DISCUSSION}

Tuberculosis is one of the diseases that cause a heavy burden to the society of India. Being one of the overpopulated countries in the world, Tuberculosis is a major threat to India. The newer techniques based on molecular studies are limited in their availability as they are expensive and requires trained personnel. So, this study aims to validate a technique that is simple, fast and less expensive. On reviewing the literature, there are only limited number of studies comparing the conventional and bleach methods of ZN with 
that of GeneXpert in TB lymphadenitis. In the present study, $9 / 30$ cases $(30 \%)$ were positive for AFB by conventional method which was higher than the study conducted by Mani Krishna and Sheetal G. Gole (20\%).[7] Annam et al.,[21] showed a $33.3 \%$ detection rate which was closest to our study.

In our study, by modified bleach method $6 / 30$ cases (20\%) were positive. The sensitivity of modified bleach method $(54.55 \%)$ was lower than the conventional method (81.81\%). The detection rate of AFB was low by modified bleach method i.e. 3 more cases were detected by conventional method. This is contrary to the findings of studies conducted by Mani Krishna et al.[7] Annam et al.,[21] and Vishal Sharma et al.,[22] in which they could detect more number of cases by modified bleach method than the conventional method of Ziehl Neelsen technique.

In our study, we observed a range of 1 to 40 bacilli per oil immersion field by conventional method. By modified bleach method we could able to make 1 to 11 bacilli per oil immersion field. The number of bacilli per field was lower by modified bleach method and bacilli tend to clump, this effect explained by centrifugation of the sample. In addition to this, Sodium hypochlorite lyses the cells to give a clear background facilitating an easy identification of bacilli. The study by Mulialem Tadesse et al.,[23] detected $26 \%$ of AFB by smear microscopy and had $27.8 \%$ sensitivity which was similar to our study.

One case that showed granuloma, necrosis in FNAC and stained positive for AFB in both the methods was not found to be positive by CBNAAT. In our study more AFB positive cases were identified in Suppurative lymphadenitis compared to the typical caseating lymphadenitis. This may be because of the immunological mechanism of the individual. Immunocompetent individuals tend to contain the TB bacilli and there are more number of granulomas but in Suppurative lymphadenitis there are more number of bacilli without granuloma formation.

\section{CONCLUSIONS}

Conventional ZN method is more sensitive than modified bleach method. AFB bacilli are readily picked up by conventional ZN method and numbers of bacilli per oil immersion method are more compared to the modified bleach method. Numbers of AFB positive cases were more among suppurative lymphadenitis. As false negative cases by CBNAAT are also reported, CBNAAT negativity does not always rule out TB. The results should be correlated with cytology and microbiology studies.

\section{ACKNOWLEDGEMENT}

We thank Indian Council of Medical research [ICMR] and the Department of Pathology, Government Vellore Medical College for their support.

\section{REFERENCES}

[1] Chakraborty AK. Epidemiology of tuberculosis: current status in India. Indian J Med Res 2004;120(4):248-76.

[2] Annual status report. TB India 2016. Revised National TB control programme.

[3] Bhatia AS, Kumar S, Harinath BC. Immunodiagnosis of tuberculosis: an update. Ind J Clin Biochem 2003;18(2):1-5.

[4] Miller FJW. Tuberculosis in children: evolution, epidemiology, treatment and prevention. New Delhi: Churchill Livingstone 1994: p. 3-17.

[5] Wares F, Balasubramanian R, Mohan A, et al. Extra pulmonary tuberculosis, management and control. In: Fraser W, Balasubramanian R, Mohan A, et al. eds. Tuberculosis Control in India. Elsevier 2005: p. 95-114.

[6] Salvador F, Los-Arcos I, Sanchez-Montalva A, et al. Epidemiology and diagnosis of tuberculous lymphadenitis in a tuberculosis low-burden country. Medicine (Baltimore) 2015;94(4):e509.

[7] Krishna M, Gole SG. Comparisons of conventional Ziehl Neelsen method of Acid fast bacilli with modified bleach method in Tuberculous lymphadenits. J Cytol 2017;34(4):188-92.

[8] Das B, Basumatari S. Incidence of Tuberculosis in cervical lymphadenopathy. A clinico -epidemiological study. International Journal of Contemporary Medical Research 2017;4(6):1254-7.

[9] Chen YM, Lee PY, Su WJ, et al. Lymph node tuberculosis: 7- year experience in Veterans General hospital, Taipei, Taiwan. Tubercle Lung Dis 1992;73(6):368-71.

[10] Ellison E, Lapuerta P, Martin SE. Fine needle aspiration diagnosis of mycobacterium lymphadenitis. Sensitivity and predictive value in the United States. Acta Cytol 1999;43(2):153-7.

[11] Wright CA, van der Burg M, Geiger D, et al. Diagnosing mycobacterium lymphadenitis in children using fine needle aspiration biopsy: cytomorphology, ZN staining and auto fluorescence-making more or less. Diagn Cytopathol 2008;36(4):245-51.

[12] Angeby K. Tuberculosis: diagnosis and drug susceptibility testing where resources are scarce. Stockholm, Karolinska University Press 2004.

[13] Skoog L Lowhagen T, Tani E. Lymphnodes. In: Gray W, edr. Diagnostic cytopathology. $1^{\text {st }}$ edn. Edinburgh: Churchill Livingstone 1999: p. 481-525.

[14] Daniel TM. Rapid diagnosis of Tuberculosis: laboratory techniques applicable in developing countries. Rev Infect Dis 1989;11(Suppl 2):S471-8.

[15] Balows A, Hausler WJ, Hermaann KL, et al. Manual of clinical microbiology. $5^{\text {th }}$ edn. Washington DC: American Society for Microbiology 1991: p. 308-11.

[16] Miliauskas J. Lymphnodes. In: Orell SR, Sterrett GF, eds. Fine Needle Aspiration Cytology. $5^{\text {th }}$ edn. New Delhi: Churchill Livingstone/ Elsevier 2012: p. 77-117.

[17] Kanungo R. Textbook of Microbiology. $10^{\text {th }}$ edn. Hyderabad: Universities Press (India) Private Limited., 2017.

[18] Agarwal M, Bajaj A, Bhatia V, et al. Comparative study of Gene Xpert with ZN stain and culture in samples of suspected pulmonary Tuberculosis. J Clin Diag Res 2016;10(5):DC09-12. 
[19] Roadmap for rolling out Xpert MTB/RIF for rapid diagnosis of TB and MDR - TB, WHO, Geneva: 2010.

[20] Central TB Division, Directorate General of Health services, Ministry of Health and Family Welfare, Government of India. Technical and Operational Guidelines for Tuberculosis control. New Delhi, India: Ministry of Health and Family Welfare, 2005.

[21] Annam V. Karigoudar MH. Yelikar BR. Improved microscopical detection of acid fast bacilli by the modified bleach method in lymphnode aspirates. Indian J Pathol Microbiol 2009;52(3):349-52.
[22] Sharma V, Sharma A. A study of modified bleach method in comparison to direct Ziehl Neelsen staining for diagnosis of pulmonary and extrapulmonary Tuberculosis in a tertiary care hospital. Int J Med Res Prof 2017;3(6):75-7.

[23] Tadasse M, Abebe G, Abdissa K, et al. GeneXpert MTB/RIF assay for the diagnosis of tuberculous lymphadenitis on concentrated fine needle aspirates in high tuberculosis burden settings. PLoS One 2015;10(9):e0137471. 